\title{
Observation of New Charmless Decays of Bottom Hadrons
}

T. Aaltonen, ${ }^{24}$ J. Adelman, ${ }^{14}$ T. Akimoto, ${ }^{56}$ B. Álvarez González, ${ }^{12, \mathrm{r}}$ S. Amerio, ${ }^{44 \mathrm{~b}, 44 \mathrm{a}}$ D. Amidei, ${ }^{35}$ A. Anastassov,${ }^{39}$ A. Annovi ${ }^{20}$ J. Antos, ${ }^{15}$ G. Apollinari,${ }^{18}$ A. Apresyan, ${ }^{49}$ T. Arisawa, ${ }^{58}$ A. Artikov, ${ }^{16}$ W. Ashmanskas,${ }^{18}$ A. Attal, ${ }^{4}$ A. Aurisano, ${ }^{54}$ F. Azfar, ${ }^{43}$ P. Azzurri, ${ }^{47 \mathrm{~d}, 47 \mathrm{a}}$ W. Badgett, ${ }^{18}$ A. Barbaro-Galtieri, ${ }^{29}$ V. E. Barnes, ${ }^{49}$ B. A. Barnett, ${ }^{26}$ V. Bartsch, ${ }^{31}$ G. Bauer, ${ }^{33}$ P.-H. Beauchemin, ${ }^{34}$ F. Bedeschi, ${ }^{47 a}$ D. Beecher,${ }^{31}$ S. Behari, ${ }^{26}$ G. Bellettini, ${ }^{47 b, 47 a}$ J. Bellinger, ${ }^{60}$ D. Benjamin, ${ }^{17}$ A. Beretvas, ${ }^{18}$ J. Beringer, ${ }^{29}$ A. Bhatti, ${ }^{51}$ M. Binkley, ${ }^{18}$ D. Bisello, ${ }^{44 b, 44 a}$ I. Bizjak,${ }^{31, w}$ R. E. Blair, ${ }^{2}$ C. Blocker, ${ }^{7}$ B. Blumenfeld, ${ }^{26}$ A. Bocci,${ }^{17}$ A. Bodek,${ }^{50}$ V. Boisvert, ${ }^{50}$ G. Bolla, ${ }^{49}$ D. Bortoletto, ${ }^{49}$ J. Boudreau, ${ }^{48}$ A. Boveia, ${ }^{11}$ B. Brau, ${ }^{11, b}$ A. Bridgeman, ${ }^{25}$ L. Brigliadori, ${ }^{44 a}$ C. Bromberg, ${ }^{36}$ E. Brubaker, ${ }^{14}$ J. Budagov, ${ }^{16}$ H. S. Budd, ${ }^{50}$ S. Budd, ${ }^{25}$ S. Burke,${ }^{18}$ K. Burkett, ${ }^{18}$ G. Busetto, ${ }^{44 b, 44 a}$ P. Bussey,${ }^{22}$ A. Buzatu, ${ }^{34}$ K. L. Byrum, ${ }^{2}$ S. Cabrera, ${ }^{17, t}$ C. Calancha, ${ }^{32}$ M. Campanelli, ${ }^{36}$ M. Campbell, ${ }^{35}$ F. Canelli, ${ }^{14,18}$ A. Canepa,${ }^{46}$ B. Carls,${ }^{25}$ D. Carlsmith,${ }^{60}$ R. Carosi, ${ }^{47 a}$ S. Carrillo, ${ }^{19, \mathrm{~m}}$ S. Carron, ${ }^{34}$ B. Casal, ${ }^{12}$ M. Casarsa, ${ }^{18}$ A. Castro,${ }^{6 b, 6 a}$ P. Catastini,${ }^{47 c, 47 a}$ D. Cauz, ${ }^{55 b, 55 a}$ V. Cavaliere, ${ }^{47 c, 47 a}$ M. Cavalli-Sforza, ${ }^{4}$ A. Cerri, ${ }^{29}$ L. Cerrito,${ }^{31, n}$ S. H. Chang, ${ }^{28}$ Y. C. Chen, ${ }^{1}$ M. Chertok,${ }^{8}$ G. Chiarelli, ${ }^{47 a}$ G. Chlachidze,${ }^{18}$ F. Chlebana, ${ }^{18}$ K. Cho, ${ }^{28}$ D. Chokheli, ${ }^{16}$ J. P. Chou, ${ }^{23}$ G. Choudalakis, ${ }^{33}$ S. H. Chuang, ${ }^{53}$ K. Chung, ${ }^{13}$ W. H. Chung, ${ }^{60}$ Y. S. Chung, ${ }^{50}$ T. Chwalek,${ }^{27}$ C. I. Ciobanu, ${ }^{45}$ M. A. Ciocci, ${ }^{47 c, 47 a}$ A. Clark, ${ }^{21}$ D. Clark,${ }^{7}$ G. Compostella, ${ }^{44 a}$ M. E. Convery, ${ }^{18}$ J. Conway, ${ }^{8}$ M. Cordelli, ${ }^{20}$ G. Cortiana, ${ }^{44 b, 44 a}$ C. A. Cox,${ }^{8}$ D. J. Cox,${ }^{8}$ F. Crescioli, ${ }^{47 b, 47 a}$

C. Cuenca Almenar, ${ }^{8, \mathrm{t}} \mathrm{J}$. Cuevas, ${ }^{12, \mathrm{r}}$ R. Culbertson, ${ }^{18}$ J. C. Cully, ${ }^{35}$ D. Dagenhart, ${ }^{18}$ M. Datta, ${ }^{18}$ T. Davies, ${ }^{22}$ P. de Barbaro, ${ }^{50}$ S. De Cecco, ${ }^{52 a}$ A. Deisher ${ }^{29}$ G. De Lorenzo, ${ }^{4}$ M. Dell'Orso, ${ }^{47 b, 47 a}$ C. Deluca, ${ }^{4}$ L. Demortier,${ }^{51}$ J. Deng, ${ }^{17}$ M. Deninno, ${ }^{6 a}$ P. F. Derwent, ${ }^{18}$ G. P. di Giovanni, ${ }^{45}$ C. Dionisi, ${ }^{52 b, 52 a}$ B. Di Ruzza, ${ }^{55 b, 55 a}$ J. R. Dittmann, ${ }^{5}$ M. D'Onofrio, ${ }^{4}$ S. Donati, ${ }^{47 b, 47 a}$ P. Dong, ${ }^{9}$ J. Donini, ${ }^{44 a}$ T. Dorigo, ${ }^{44 a}$ S. Dube,${ }^{53}$ J. Efron, ${ }^{40}$ A. Elagin, ${ }^{54}$ R. Erbacher, ${ }^{8}$ D. Errede, ${ }^{25}$ S. Errede,${ }^{25}$ R. Eusebi, ${ }^{18}$ H. C. Fang, ${ }^{29}$ S. Farrington, ${ }^{43}$ W. T. Fedorko, ${ }^{14}$ R. G. Feild, ${ }^{61}$ M. Feindt, ${ }^{27}$ J. P. Fernandez,${ }^{32}$ C. Ferrazza, ${ }^{47 d, 47 a}$ R. Field, ${ }^{19}$ G. Flanagan,${ }^{49}$ R. Forrest,${ }^{8}$ M. J. Frank, ${ }^{5}$ M. Franklin, ${ }^{23}$ J. C. Freeman, ${ }^{18}$ I. Furic, ${ }^{19}$ M. Gallinaro, ${ }^{52 a}$ J. Galyardt, ${ }^{13}$ F. Garberson, ${ }^{11}$ J. E. Garcia, ${ }^{21}$ A. F. Garfinkel, ${ }^{49}$ K. Genser,${ }^{18}$ H. Gerberich, ${ }^{25}$ D. Gerdes,${ }^{35}$ A. Gessler, ${ }^{27}$ S. Giagu, ${ }^{52 b, 52 a}$ V. Giakoumopoulou, ${ }^{3}$ P. Giannetti, ${ }^{47 a}$ K. Gibson, ${ }^{48}$ J. L. Gimmell, ${ }^{50}$ C. M. Ginsburg, ${ }^{18}$ N. Giokaris, ${ }^{3}$ M. Giordani, ${ }^{55 b, 55 a}$ P. Giromini, ${ }^{20}$ M. Giunta, ${ }^{47 b, 47 a}$ G. Giurgiu, ${ }^{26}$ V. Glagolev, ${ }^{16}$ D. Glenzinski, ${ }^{18}$ M. Gold, ${ }^{38}$ N. Goldschmidt, ${ }^{19}$ A. Golossanov, ${ }^{18}$ G. Gomez, ${ }^{12}$ G. Gomez-Ceballos,${ }^{33}$ M. Goncharov, ${ }^{33}$ O. González, ${ }^{32}$ I. Gorelov, ${ }^{38}$ A. T. Goshaw, ${ }^{17}$ K. Goulianos, ${ }^{51}$ A. Gresele, ${ }^{44 b, 44 a}$ S. Grinstein, ${ }^{23}$ C. Grosso-Pilcher,${ }^{14}$ R. C. Group, ${ }^{18}$ U. Grundler, ${ }^{25}$ J. Guimaraes da Costa, ${ }^{23}$ Z. Gunay-Unalan, ${ }^{36}$ C. Haber, ${ }^{29}$ K. Hahn, ${ }^{33}$ S. R. Hahn, ${ }^{18}$ E. Halkiadakis, ${ }^{53}$ B.-Y. Han ${ }^{50}$ J. Y. Han, ${ }^{50}$ F. Happacher, ${ }^{20}$ K. Hara ${ }^{56}$ D. Hare,${ }^{53}$ M. Hare, ${ }^{57}$ S. Harper, ${ }^{43}$ R. F. Harr, ${ }^{59}$ R. M. Harris, ${ }^{18}$ M. Hartz, ${ }^{48}$ K. Hatakeyama, ${ }^{51}$ C. Hays, ${ }^{43}$ M. Heck, ${ }^{27}$ A. Heijboer, ${ }^{46}$ J. Heinrich, ${ }^{46}$ C. Henderson, ${ }^{33}$ M. Herndon, ${ }^{60}$ J. Heuser, ${ }^{27}$ S. Hewamanage, ${ }^{5}$ D. Hidas, ${ }^{17}$ C. S. Hill, ${ }^{11, d}$ D. Hirschbuehl,${ }^{27}$ A. Hocker, ${ }^{18}$ S. Hou, ${ }^{1}$ M. Houlden, ${ }^{30}$ S.-C. Hsu, ${ }^{29}$

B. T. Huffman, ${ }^{43}$ R. E. Hughes, ${ }^{40}$ U. Husemann, ${ }^{61}$ M. Hussein, ${ }^{36}$ J. Huston, ${ }^{36}$ J. Incandela, ${ }^{11}$ G. Introzzi,${ }^{47 a}$ M. Iori, ${ }^{52 b, 52 a}$ A. Ivanov, ${ }^{8}$ E. James, ${ }^{18}$ D. Jang, ${ }^{13}$ B. Jayatilaka, ${ }^{17}$ E. J. Jeon, ${ }^{28}$ M. K. Jha ${ }^{6 a}$ S. Jindariani, ${ }^{18}$ W. Johnson, ${ }^{8}$ M. Jones ${ }^{49}$ K. K. Joo, ${ }^{28}$ S. Y. Jun, ${ }^{13}$ J.E. Jung, ${ }^{28}$ T. R. Junk, ${ }^{18}$ T. Kamon,${ }^{54}$ D. Kar, ${ }^{19}$ P. E. Karchin,${ }^{59}$ Y. Kato, ${ }^{42}$ R. Kephart, ${ }^{18}$

J. Keung, ${ }^{46}$ V. Khotilovich, ${ }^{54}$ B. Kilminster, ${ }^{18}$ D. H. Kim, ${ }^{28}$ H. S. Kim, ${ }^{28}$ H. W. Kim, ${ }^{28}$ J.E. Kim, ${ }^{28}$ M. J. Kim, ${ }^{20}$ S. B. Kim ${ }^{28}$ S. H. Kim,${ }^{56}$ Y. K. Kim, ${ }^{14}$ N. Kimura ${ }^{56}$ L. Kirsch, ${ }^{7}$ S. Klimenko, ${ }^{19}$ B. Knuteson, ${ }^{33}$ B. R. Ko, ${ }^{17}$ K. Kondo, ${ }^{58}$ D. J. Kong, ${ }^{28}$ J. Konigsberg, ${ }^{19}$ A. Korytov, ${ }^{19}$ A. V. Kotwal, ${ }^{17}$ M. Kreps,${ }^{27}$ J. Kroll,${ }^{46}$ D. Krop,${ }^{14}$ N. Krumnack,${ }^{5}$ M. Kruse, ${ }^{17}$ V. Krutelyov, ${ }^{11}$ T. Kubo, ${ }^{56}$ T. Kuhr, ${ }^{27}$ N. P. Kulkarni ${ }^{59}$ M. Kurata, ${ }^{56}$ S. Kwang, ${ }^{14}$ A. T. Laasanen, ${ }^{49}$ S. Lami,${ }^{47 a}$ S. Lammel, ${ }^{18}$ M. Lancaster, ${ }^{31}$ R. L. Lander, ${ }^{8}$ K. Lannon, ${ }^{40, \mathrm{q}}$ A. Lath, ${ }^{53}$ G. Latino, ${ }^{47 c, 47 a}$ I. Lazzizzera, ${ }^{44 b, 44 a}$ T. LeCompte, ${ }^{2}$ E. Lee, ${ }^{54}$ H. S. Lee, ${ }^{14}$ S. W. Lee, ${ }^{54, s}$ S. Leone, ${ }^{47 a}$ J. D. Lewis, ${ }^{18}$ C.-S. Lin, ${ }^{29}$ J. Linacre, ${ }^{43}$ M. Lindgren, ${ }^{18}$ E. Lipeles, ${ }^{46}$

A. Lister, ${ }^{8}$ D. O. Litvintsev, ${ }^{18}$ C. Liu, ${ }^{48}$ T. Liu, ${ }^{18}$ N. S. Lockyer, ${ }^{46}$ A. Loginov, ${ }^{61}$ M. Loreti, ${ }^{44 b, 44 a}$ L. Lovas, ${ }^{15}$

D. Lucchesi ${ }^{44 b, 44 a}$ C. Luci, ${ }^{52 b, 52 a}$ J. Lueck,${ }^{27}$ P. Lujan, ${ }^{29}$ P. Lukens, ${ }^{18}$ G. Lungu, ${ }^{51}$ L. Lyons,${ }^{43}$ J. Lys, ${ }^{29}$ R. Lysak,${ }^{15}$ D. MacQueen, ${ }^{34}$ R. Madrak, ${ }^{18}$ K. Maeshima, ${ }^{18}$ K. Makhoul, ${ }^{33}$ T. Maki,${ }^{24}$ P. Maksimovic, ${ }^{26}$ S. Malde,${ }^{43}$ S. Malik,${ }^{31}$ G. Manca, ${ }^{30, f}$ A. Manousakis-Katsikakis, ${ }^{3}$ F. Margaroli, ${ }^{49}$ C. Marino, ${ }^{27}$ C. P. Marino, ${ }^{25}$ A. Martin, ${ }^{61}$ V. Martin, ${ }^{22,1}$ M. Martínez, ${ }^{4}$ R. Martínez-Ballarín, ${ }^{32}$ T. Maruyama, ${ }^{56}$ P. Mastrandrea, ${ }^{52 a}$ T. Masubuchi, ${ }^{56}$ M. Mathis,${ }^{26}$ M. E. Mattson, ${ }^{59}$ P. Mazzanti, ${ }^{6 a}$ K. S. McFarland,${ }^{50}$ P. McIntyre, ${ }^{54}$ R. McNulty, ${ }^{30, k}$ A. Mehta,${ }^{30}$ P. Mehtala, ${ }^{24}$ A. Menzione,${ }^{47 a}$ P. Merkel, ${ }^{49}$

C. Mesropian ${ }^{51}$ T. Miao, ${ }^{18}$ N. Miladinovic, ${ }^{7}$ R. Miller, ${ }^{36}$ C. Mills,${ }^{23}$ M. Milnik,${ }^{27}$ A. Mitra, ${ }^{1}$ G. Mitselmakher, ${ }^{19}$

H. Miyake, ${ }^{56}$ N. Moggi, ${ }^{6 a}$ C. S. Moon, ${ }^{28}$ R. Moore,${ }^{18}$ M. J. Morello, ${ }^{47 b, 47 a}$ J. Morlock, ${ }^{27}$ P. Movilla Fernandez, ${ }^{18}$ J. Mülmenstädtt, ${ }^{29}$ A. Mukherjee, ${ }^{18}$ Th. Muller, ${ }^{27}$ R. Mumford, ${ }^{26}$ P. Murat, ${ }^{18}$ M. Mussini, ${ }^{6 b, 6 a}$ J. Nachtman, ${ }^{18}$ Y. Nagai, ${ }^{56}$ A. Nagano, ${ }^{56}$ J. Naganoma, ${ }^{56}$ K. Nakamura, ${ }^{56}$ I. Nakano, ${ }^{41}$ A. Napier,${ }^{57}$ V. Necula, ${ }^{17}$ J. Nett,${ }^{60}$ C. Neu, ${ }^{46, u}$ 
M. S. Neubauer, ${ }^{25}$ S. Neubauer, ${ }^{27}$ J. Nielsen,${ }^{29, \mathrm{~h}}$ L. Nodulman, ${ }^{2}$ M. Norman, ${ }^{10}$ O. Norniella, ${ }^{25}$ E. Nurse, ${ }^{31}$ L. Oakes, ${ }^{43}$ S. H. Oh, ${ }^{17}$ Y. D. Oh, ${ }^{28}$ I. Oksuzian, ${ }^{19}$ T. Okusawa, ${ }^{42}$ R. Orava, ${ }^{24}$ K. Osterberg, ${ }^{24}$ S. Pagan Griso, ${ }^{44 b, 44 a}$ E. Palencia ${ }^{18}$ V. Papadimitriou, ${ }^{18}$ A. Papaikonomou, ${ }^{27}$ A. A. Paramonov, ${ }^{14}$ B. Parks, ${ }^{40}$ S. Pashapour, ${ }^{34}$ J. Patrick, ${ }^{18}$ G. Pauletta, ${ }^{55 b, 55 a}$ M. Paulini, ${ }^{13}$ C. Paus,${ }^{33}$ T. Peiffer, ${ }^{27}$ D. E. Pellett, ${ }^{8}$ A. Penzo, ${ }^{55 a}$ T. J. Phillips,${ }^{17}$ G. Piacentino, ${ }^{47 a}$ E. Pianori,${ }^{46}$ L. Pinera, ${ }^{19}$ K. Pitts, ${ }^{25}$ C. Plager, ${ }^{9}$ L. Pondrom, ${ }^{60}$ O. Poukhov, ${ }^{16, a}$ N. Pounder, ${ }^{43}$ F. Prakoshyn, ${ }^{16}$ A. Pronko, ${ }^{18}$ J. Proudfoot, ${ }^{2}$ F. Ptohos, ${ }^{18, j}$ E. Pueschel, ${ }^{13}$ G. Punzi, ${ }^{47 b, 47 a}$ J. Pursley, ${ }^{60}$ J. Rademacker, ${ }^{43, d}$ A. Rahaman, ${ }^{48}$ V. Ramakrishnan, ${ }^{60}$ N. Ranjan, ${ }^{49}$ I. Redondo, ${ }^{32}$ P. Renton, ${ }^{43}$ M. Renz,${ }^{27}$ M. Rescigno, ${ }^{52 \mathrm{a}}$ S. Richter, ${ }^{27}$ F. Rimondi, ${ }^{6 \mathrm{~b}, 6 \mathrm{a}}$ L. Ristori, ${ }^{47 \mathrm{a}}$ A. Robson, ${ }^{22}$ T. Rodrigo, ${ }^{12}$ T. Rodriguez, ${ }^{46}$ E. Rogers ${ }^{25}$ S. Rolli, ${ }^{57}$ R. Roser, ${ }^{18}$ M. Rossi, ${ }^{55 a}$ R. Rossin, ${ }^{11}$ P. Roy, ${ }^{34}$ A. Ruiz, ${ }^{12}$ J. Russ, ${ }^{13}$ V. Rusu, ${ }^{18}$ H. Saarikko, ${ }^{24}$ A. Safonov, ${ }^{54}$ W. K. Sakumoto, ${ }^{50}$ O. Saltó, ${ }^{4}$ L. Santi, ${ }^{55 b, 55 a}$ S. Sarkar,${ }^{52 b, 52 a}$ L. Sartori, ${ }^{47 a}$ K. Sato, ${ }^{18}$ A. Savoy-Navarro, ${ }^{45}$ P. Schlabach,${ }^{18}$ A. Schmidt,${ }^{27}$ E. E. Schmidt, ${ }^{18}$ M. A. Schmidt, ${ }^{14}$ M. P. Schmidt, ${ }^{61, a}$ M. Schmitt, ${ }^{39}$ T. Schwarz, ${ }^{8}$ L. Scodellaro, ${ }^{12}$ A. Scribano, ${ }^{47 c, 47 a}$ F. Scuri, ${ }^{47 a}$ A. Sedov, ${ }^{49}$ S. Seidel, ${ }^{38}$ Y. Seiya ${ }^{42}$ A. Semenov, ${ }^{16}$ L. Sexton-Kennedy, ${ }^{18}$ F. Sforza, ${ }^{47 a}$ A. Sfyrla, ${ }^{25}$ S. Z. Shalhout, ${ }^{59}$ T. Shears,${ }^{30}$ P. F. Shepard, ${ }^{48}$ M. Shimojima, ${ }^{56, p}$ S. Shiraishi, ${ }^{14}$ M. Shochet, ${ }^{14}$ Y. Shon, ${ }^{60}$ I. Shreyber,${ }^{37}$ A. Sidoti, ${ }^{47 a}$ P. Sinervo, ${ }^{34}$ A. Sisakyan, ${ }^{16}$ A. J. Slaughter ${ }^{18}$ J. Slaunwhite, ${ }^{40}$ K. Sliwa,${ }^{57}$ J. R. Smith ${ }^{8}$ F. D. Snider, ${ }^{18}$ R. Snihur, ${ }^{34}$ A. Soha,${ }^{8}$ S. Somalwar, ${ }^{53}$ V. Sorin, ${ }^{36}$ J. Spalding, ${ }^{18}$ T. Spreitzer, ${ }^{34}$ P. Squillacioti, ${ }^{47 c, 47 a}$ M. Stanitzki ${ }^{61}$ R. St. Denis,${ }^{22}$ B. Stelzer, ${ }^{34}$ O. Stelzer-Chilton, ${ }^{34}$ D. Stentz, ${ }^{39}$ J. Strologas, ${ }^{38}$ G. L. Strycker, ${ }^{35}$ D. Stuart, ${ }^{11}$ J. S. Suh, ${ }^{28}$ A. Sukhanov, ${ }^{19}$ I. Suslov, ${ }^{16}$ T. Suzuki, ${ }^{56}$ A. Taffard, ${ }^{25, g}$ R. Takashima, ${ }^{41}$ Y. Takeuchi,${ }^{56}$ R. Tanaka, ${ }^{41}$ M. Tecchio, ${ }^{35}$ P. K. Teng, ${ }^{1}$ K. Terashi,${ }^{51}$ R. Tesarek, ${ }^{18}$ J. Thom,,${ }^{18, i}$

A. S. Thompson, ${ }^{22}$ G. A. Thompson, ${ }^{25}$ E. Thomson, ${ }^{46}$ P. Tipton, ${ }^{61}$ P. Ttito-Guzmán, ${ }^{32}$ S. Tkaczyk, ${ }^{18}$ D. Toback, ${ }^{54}$ S. Tokar ${ }^{15} \mathrm{~K}$. Tollefson, ${ }^{36} \mathrm{~T}$. Tomura ${ }^{56} \mathrm{D}$. Tonelli, ${ }^{18} \mathrm{~S}$. Torre,${ }^{20} \mathrm{D}$. Torretta ${ }^{18} \mathrm{P}$. Totaro, ${ }^{55 \mathrm{~b}, 55 \mathrm{a}} \mathrm{S}$. Tourneur, ${ }^{45}$ M. Trovato, ${ }^{47 a}$ S.-Y. Tsai, ${ }^{1}$ Y. Tu, ${ }^{46}$ N. Turini, ${ }^{47 c, 47 a}$ F. Ukegawa, ${ }^{56}$ S. Vallecorsa, ${ }^{21}$ N. van Remortel,,${ }^{24, c}$ A. Varganov, ${ }^{35}$ E. Vataga ${ }^{47 \mathrm{~d}, 47 \mathrm{a}}$ F. Vázquez, ${ }^{19, \mathrm{~m}} \mathrm{G}$. Velev, ${ }^{18} \mathrm{C}$. Vellidis, ${ }^{3} \mathrm{M}$. Vidal,${ }^{32} \mathrm{R}$. Vidal, ${ }^{18} \mathrm{I}$. Vila, ${ }^{12} \mathrm{R}$. Vilar, ${ }^{12} \mathrm{~T}$. Vine, ${ }^{31} \mathrm{M}$. Vogel,${ }^{38}$ I. Volobouev, ${ }^{29, \mathrm{~s}}$ G. Volpi, ${ }^{47 b, 47 a}$ P. Wagner,${ }^{46}$ R. G. Wagner, ${ }^{2}$ R. L. Wagner,${ }^{18}$ W. Wagner, ${ }^{27, v}$ J. Wagner-Kuhr, ${ }^{27}$

T. Wakisaka, ${ }^{42}$ R. Wallny, ${ }^{9}$ S. M. Wang, ${ }^{1}$ A. Warburton, ${ }^{34}$ D. Waters,${ }^{31}$ M. Weinberger, ${ }^{54}$ J. Weinelt, ${ }^{27}$ W. C. Wester III,,${ }^{18}$ B. Whitehouse, ${ }^{57}$ D. Whiteson, ${ }^{46,9}$ A. B. Wicklund, ${ }^{2}$ E. Wicklund,${ }^{18}$ S. Wilbur, ${ }^{14}$ G. Williams,${ }^{34}$ H. H. Williams, ${ }^{46}$ P. Wilson, ${ }^{18}$ B. L. Winer, ${ }^{40}$ P. Wittich, ${ }^{18, i}$ S. Wolbers, ${ }^{18}$ C. Wolfe, ${ }^{14}$ T. Wright,${ }^{35} \mathrm{X}$. Wu, ${ }^{21}$ F. Würthwein,${ }^{10}$ S. Xie, ${ }^{33}$ A. Yagil, ${ }^{10}$ K. Yamamoto, ${ }^{42}$ J. Yamaoka, ${ }^{17}$ U. K. Yang, ${ }^{14,0}$ Y. C. Yang,${ }^{28}$ W. M. Yao, ${ }^{29}$ G. P. Yeh,${ }^{18}$ J. Yoh,${ }^{18}$ K. Yorita, ${ }^{58}$ T. Yoshida ${ }^{42}$ G. B. Yu, ${ }^{50}$ I. Yu, ${ }^{28}$ S. S. Yu, ${ }^{18}$ J. C. Yun, ${ }^{18}$ L. Zanello, ${ }^{52 b, 52 a}$ A. Zanetti, ${ }^{55 a}$ X. Zhang, ${ }^{25}$ Y. Zheng, ${ }^{9, e}$ and S. Zucchelli ${ }^{6 b, 6 a}$

(CDF Collaboration)

${ }^{1}$ Institute of Physics, Academia Sinica, Taipei, Taiwan 11529, Republic of China

${ }^{2}$ Argonne National Laboratory, Argonne, Illinois 60439, USA

${ }^{3}$ University of Athens, 15771 Athens, Greece

${ }^{4}$ Institut de Fisica d'Altes Energies, Universitat Autonoma de Barcelona, E-08193, Bellaterra (Barcelona), Spain

${ }^{5}$ Baylor University, Waco, Texas 76798, USA

${ }^{6 a}$ Istituto Nazionale di Fisica Nucleare Bologna, I-40127 Bologna, Italy

${ }^{6 \mathrm{~b}}$ University of Bologna, I-40127 Bologna, Italy

${ }^{7}$ Brandeis University, Waltham, Massachusetts 02254, USA

${ }^{8}$ University of California, Davis, Davis, California 95616, USA

${ }^{9}$ University of California, Los Angeles, Los Angeles, California 90024, USA

${ }^{10}$ University of California, San Diego, La Jolla, California 92093, USA

${ }^{11}$ University of California, Santa Barbara, Santa Barbara, California 93106, USA

${ }^{12}$ Instituto de Fisica de Cantabria, CSIC-University of Cantabria, 39005 Santander, Spain

${ }^{13}$ Carnegie Mellon University, Pittsburgh, Pennsylvania 15213, USA

${ }^{14}$ Enrico Fermi Institute, University of Chicago, Chicago, Illinois 60637, USA

${ }^{15}$ Comenius University, 84248 Bratislava, Slovakia; Institute of Experimental Physics, 04001 Kosice, Slovakia

${ }^{16}$ Joint Institute for Nuclear Research, RU-141980 Dubna, Russia

${ }^{17}$ Duke University, Durham, North Carolina 27708, USA

${ }^{18}$ Fermi National Accelerator Laboratory, Batavia, Illinois 60510, USA

${ }^{19}$ University of Florida, Gainesville, Florida 32611, USA

${ }^{20}$ Laboratori Nazionali di Frascati, Istituto Nazionale di Fisica Nucleare, I-00044 Frascati, Italy

${ }^{21}$ University of Geneva, CH-1211 Geneva 4, Switzerland

${ }^{22}$ Glasgow University, Glasgow G12 8QQ, United Kingdom

${ }^{23}$ Harvard University, Cambridge, Massachusetts 02138, USA 
${ }^{24}$ Division of High Energy Physics, Department of Physics, University of Helsinki and Helsinki Institute of Physics, FIN-00014, Helsinki, Finland

${ }^{25}$ University of Illinois, Urbana, Illinois 61801, USA

${ }^{26}$ The Johns Hopkins University, Baltimore, Maryland 21218, USA

${ }^{27}$ Institut für Experimentelle Kernphysik, Universität Karlsruhe, 76128 Karlsruhe, Germany

${ }^{28}$ Center for High Energy Physics: Kyungpook National University, Daegu 702-701, Korea; Seoul National University, Seoul 151-742, Korea; Sungkyunkwan University, Suwon 440-746, Korea;

Korea Institute of Science and Technology Information, Daejeon, 305-806, Korea; Chonnam National University, Gwangju, 500-757, Korea

${ }^{29}$ Ernest Orlando Lawrence Berkeley National Laboratory, Berkeley, California 94720, USA

${ }^{30}$ University of Liverpool, Liverpool L69 7ZE, United Kingdom

${ }^{31}$ University College London, London WC1E 6BT, United Kingdom

${ }^{32}$ Centro de Investigaciones Energeticas Medioambientales y Tecnologicas, E-28040 Madrid, Spain

${ }^{33}$ Massachusetts Institute of Technology, Cambridge, Massachusetts 02139

${ }^{34}$ Institute of Particle Physics: McGill University, Montréal, Québec, Canada H3A 2T8;

Simon Fraser University, Burnaby, British Columbia, Canada V5A 1S6; University of Toronto, Toronto, Ontario, Canada M5S 1A7; and TRIUMF, Vancouver, British Columbia, Canada V6T $2 A 3$

${ }^{35}$ University of Michigan, Ann Arbor, Michigan 48109, USA

${ }^{36}$ Michigan State University, East Lansing, Michigan 48824, USA

${ }^{37}$ Institution for Theoretical and Experimental Physics, ITEP, Moscow 117259, Russia

${ }^{38}$ University of New Mexico, Albuquerque, New Mexico 87131, USA

${ }^{39}$ Northwestern University, Evanston, Illinois 60208, USA

${ }^{40}$ The Ohio State University, Columbus, Ohio 43210, USA

${ }^{41}$ Okayama University, Okayama 700-8530, Japan

${ }^{42}$ Osaka City University, Osaka 588, Japan

${ }^{43}$ University of Oxford, Oxford OX1 3RH, United Kingdom

${ }^{44 a}$ Istituto Nazionale di Fisica Nucleare, Sezione di Padova-Trento, I-35131 Padova, Italy

${ }^{44 b}$ University of Padova, I-35131 Padova, Italy

${ }^{45}$ LPNHE, Universite Pierre et Marie Curie/IN2P3-CNRS, UMR7585, Paris, F-75252 France

${ }^{46}$ University of Pennsylvania, Philadelphia, Pennsylvania 19104, USA

${ }^{47 a}$ Istituto Nazionale di Fisica Nucleare Pisa, I-56127 Pisa, Italy

${ }^{47 \mathrm{~b}}$ University of Pisa, I-56127 Pisa, Italy

${ }^{47 c}$ University of Siena, I-56127 Pisa, Italy

${ }^{47 \mathrm{~d}}$ Scuola Normale Superiore, I-56127 Pisa, Italy

${ }^{48}$ University of Pittsburgh, Pittsburgh, Pennsylvania 15260, USA

${ }^{49}$ Purdue University, West Lafayette, Indiana 47907, USA

${ }^{50}$ University of Rochester, Rochester, New York 14627, USA

${ }^{51}$ The Rockefeller University, New York, New York 10021, USA

${ }^{52 \mathrm{a}}$ Istituto Nazionale di Fisica Nucleare, Sezione di Roma 1, I-00185 Roma, Italy

${ }^{52 \mathrm{~b}}$ Sapienza Università di Roma, I-00185 Roma, Italy

${ }^{53}$ Rutgers University, Piscataway, New Jersey 08855, USA

${ }^{54}$ Texas A\&M University, College Station, Texas 77843, USA

${ }^{55 \mathrm{a}}$ Istituto Nazionale di Fisica Nucleare Trieste/Udine, I-34100 Trieste, Italy

${ }^{55 \mathrm{~b}}$ University of Trieste/Udine, I-33100 Udine, Italy

${ }^{56}$ University of Tsukuba, Tsukuba, Ibaraki 305, Japan

${ }^{57}$ Tufts University, Medford, Massachusetts 02155, USA

${ }^{58}$ Waseda University, Tokyo 169, Japan

${ }^{59}$ Wayne State University, Detroit, Michigan 48201, USA

${ }^{60}$ University of Wisconsin, Madison, Wisconsin 53706, USA

${ }^{61}$ Yale University, New Haven, Connecticut 06520, USA

(Received 22 December 2008; published 17 July 2009)

We search for new charmless decays of neutral $b$ hadrons to pairs of charged hadrons, using $1 \mathrm{fb}^{-1}$ of data collected by the CDF II detector at the Fermilab Tevatron. We report the first observation of the $B_{s}^{0} \rightarrow K^{-} \pi^{+}$decay and measure $\mathcal{B}\left(B_{s}^{0} \rightarrow K^{-} \pi^{+}\right)=(5.0 \pm 0.7$ (stat) \pm 0.8 (syst $\left.)\right) \times 10^{-6}$. We also report the first observation of charmless $b$-baryon decays, and measure $\mathcal{B}\left(\Lambda_{b}^{0} \rightarrow p \pi^{-}\right)=(3.5 \pm 0.6$ (stat $) \pm$ 0.9 (syst) $) \times 10^{-6}$ and $\mathcal{B}\left(\Lambda_{b}^{0} \rightarrow p K^{-}\right)=(5.6 \pm 0.8$ (stat) \pm 1.5 (syst $) \times 10^{-6}$. No evidence is found for other modes, and we set the limit $\mathcal{B}\left(B_{s}^{0} \rightarrow \pi^{+} \pi^{-}\right)<1.2 \times 10^{-6}$ at $90 \%$ C.L. 
Two-body nonleptonic charmless decays of $b$ hadrons are among the most widely studied processes in flavor physics. The variety of open channels involving similar final states provides crucial experimental information to improve the accuracy of effective models of strong interaction dynamics. The quark-level transition $b \rightarrow u$ makes decay amplitudes sensitive to $\gamma$, the least known angle of the quark-mixing (Cabibbo-Kobayashi-Maskawa, CKM) matrix. Significant contributions from higher-order ("penguin") transitions provide sensitivity to the possible presence of new physics in internal loops, if the observed decay rates are inconsistent with expectations.

Rich experimental data are currently available for $B^{+}$ and $B^{0}$ mesons, produced in large quantities in $\Upsilon(4 S)$ decays [1], while much less is experimentally known about the charmless decay modes of the $B_{s}^{0}$, which are expected to exhibit an equally rich phenomenology. Information from $B_{s}^{0}$ decays is needed to better constrain the phenomenological models of hadronic amplitudes in heavy flavor decays. This would lead to increased precision in comparing data to predictions, allowing extraction of CKM parameters from non-tree-level amplitudes [2] and greater sensitivity to new physics contributions.

Of the possible $B_{s}^{0}$ decay modes into pairs of charmless pseudoscalar mesons, only $B_{s}^{0} \rightarrow K^{+} K^{-}$has been observed to date [3]. The $B_{s}^{0} \rightarrow K^{-} \pi^{+}$mode is of particular interest, because its branching fraction is sensitive to the CKM angle $\gamma$ [4] and the current experimental bound [3] is lower than most predictions [5-7].

A measurement of the branching fraction of the $B_{s}^{0} \rightarrow$ $\pi^{+} \pi^{-}$mode, along with the $B^{0} \rightarrow K^{+} K^{-}$mode, would allow a determination of the strength of penguinannihilation amplitudes [8], which is currently poorly known and a source of significant uncertainty in many calculations [6]. The present search is sensitive to both modes. Two-body charmless decays are also expected from bottom baryons. The modes $\Lambda_{b}^{0} \rightarrow p K^{-}$and $\Lambda_{b}^{0} \rightarrow p \pi^{-}$ are predicted to have measurable branching fractions, of order $10^{-6}$ [9], and, in addition to the interest in their observation, must be considered as a possible background to the rare $B_{s}^{0}$ and $B^{0}$ modes being investigated.

In this Letter we report the results of a search for rare decays of neutral bottom hadrons into a pair of charged charmless hadrons ( $p, K$, or $\pi$ ), performed in $1 \mathrm{fb}^{-1}$ of $\bar{p} p$ collisions at $\sqrt{s}=1.96 \mathrm{TeV}$, collected by the upgraded Collider Detector (CDF II) at the Fermilab Tevatron. We report the first observation of modes $B_{s}^{0} \rightarrow K^{-} \pi^{+}, \Lambda_{b}^{0} \rightarrow$ $p K^{-}$, and $\Lambda_{b}^{0} \rightarrow p \pi^{-}$, and measure their relative branching fractions [10].

CDF II is a multipurpose magnetic spectrometer surrounded by calorimeters and muon detectors $[11,12]$. The resolution on transverse momentum of charged particles is
$\sigma_{p_{T}} / p_{T}^{2} \sim 0.15 \% /(\mathrm{GeV} / c)$, corresponding to a typical mass resolution of $22 \mathrm{MeV} / c^{2}$ for our signals. The specific ionization energy loss $(d E / d x)$ of charged particles can be measured from the charge collected by the drift chamber $(\mathrm{COT})$, and provides $1.5 \sigma$ separation between kaons and pions with momenta greater than $2 \mathrm{GeV} / c$. The data were collected by a three-level trigger system [13], using a set of requirements specifically aimed at selecting two-pronged $B$ decays [3]. Two opposite-charge particles are required, with reconstructed transverse momenta $p_{T 1}, p_{T 2}>$ $2 \mathrm{GeV} / c$, the scalar sum $p_{T 1}+p_{T 2}>5.5 \mathrm{GeV} / c$, and an azimuthal opening angle $\Delta \phi<135^{\circ}$. The impact parameter $d$ (distance of closest approach to the beam line) of the two tracks is required to be $0.1<d<1.0 \mathrm{~mm}$, reducing the light-quark background by 2 orders of magnitude while preserving about half of the signal. An openingangle requirement $20^{\circ}<\Delta \phi<135^{\circ}$, preferentially selects two-body $B$ decays over multibody decays with $97 \%$ efficiency and further reduces background. Each track pair is then used to form a $B$ candidate, which is required to have an impact parameter $d_{B}<140 \mu \mathrm{m}$ and to have traveled a distance $L_{T}>200 \mu \mathrm{m}$ in the transverse plane. The overall acceptance of the trigger selection is $\approx 2 \%$ for $b$ hadrons with $p_{T}>4 \mathrm{GeV} / c$ and $|\eta|<1$.

The offline selection is based on a more accurate determination of the same quantities used in the trigger, with the addition of two further observables: the isolation $\left(I_{B}\right)$ of the $B$ candidate [14], and the quality of the three-dimensional fit ( $\chi^{2}$ with 1 d.o.f.) of the decay vertex of the $B$ candidate. Requiring a large value of $I_{B}$ reduces the background from light-quark jets, and a low $\chi^{2}$ reduces the background from decays of different long-lived particles within the event, owing to the good resolution of the SVX detector in the $z$ direction. The selection is optimized for detection of the $B_{s}^{0} \rightarrow K^{-} \pi^{+}$mode. Maximal sensitivity for both discovery and limit setting is achieved with a single choice of selection requirements [15] by minimizing the variance of the estimate of the branching fraction in the absence of signal [16]. The variance is evaluated by performing the full measurement procedure on simulated samples containing background and all signals from the known modes, but no $B_{s}^{0} \rightarrow K^{-} \pi^{+}$signal. The background fraction for each selection is determined from data by extrapolating the mass sidebands of the signal, and the signal yield is predicted by a detailed detector simulation. This procedure yields the final selection: $I_{B}>0.525, \chi^{2}<5, d>$ $120 \mu \mathrm{m}, d_{B}<60 \mu \mathrm{m}$, and $L_{T}>350 \mu \mathrm{m}$.

No more than one $B$ candidate per event is found after this selection, and a mass $\left(m_{\pi \pi}\right)$ is assigned to each, using a charged pion mass assignment for both decay products. The resulting mass distribution is shown in Fig. 1. A large peak is visible, dominated by the overlapping contributions 


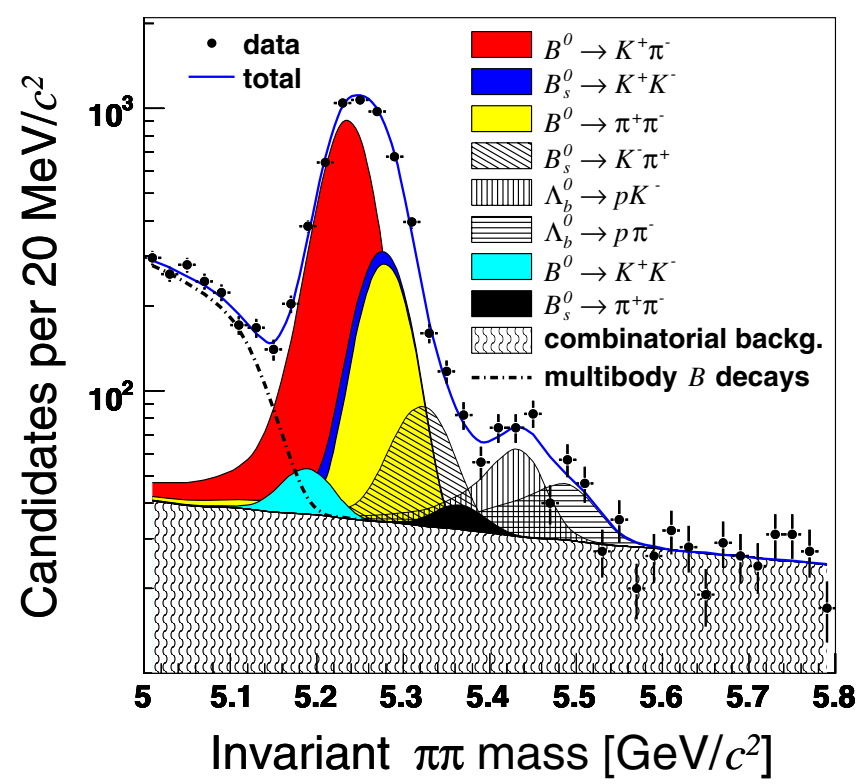

FIG. 1 (color online). Mass distribution of the 8286 reconstructed candidates. The charged pion mass is assigned to both tracks. The total projection and projections of each signal and background component of the likelihood fit are overlaid on the data distribution. Signals and multibody $B$ background components are shown stacked on the combinatorial background component.

of the $B^{0} \rightarrow K^{+} \pi^{-}, \quad B^{0} \rightarrow \pi^{+} \pi^{-}$, and $B_{s}^{0} \rightarrow K^{+} K^{-}$ modes as seen in Ref. [3]. A $B^{0} \rightarrow K^{+} K^{-}$signal would appear as an enhancement around $5.18 \mathrm{GeV} / c^{2}$, while signals for the other modes of this search are expected at masses higher than the main peak $\left(5.33-5.55 \mathrm{GeV} / c^{2}\right)$. Backgrounds include misreconstructed multibody $b$-hadron decays (physics background) and random pairs of charged particles (combinatorial background).

We used an unbinned likelihood fit, incorporating kinematic (kin) and particle identification (PID) information, to determine the fraction of each individual mode in our sample. The likelihood for the $i$ th event is

$$
\begin{aligned}
\mathcal{L}_{i}= & (1-b) \sum_{j} f_{j} \mathcal{L}_{j}^{\mathrm{kin}} \mathcal{L}_{j}^{\mathrm{PID}}+b\left(f_{p} \mathcal{L}_{p}^{\mathrm{kin}} \mathcal{L}_{p}^{\mathrm{PID}}\right. \\
& \left.+\left(1-f_{p}\right) \mathcal{L}_{c}^{\mathrm{kin}} \mathcal{L}_{c}^{\mathrm{PID}}\right),
\end{aligned}
$$

where the index $j$ runs over all signal modes, and the index " $p$ " (" $c$ ") labels the physics (combinatorial) background terms. The $f_{j}$ are the signal fractions to be determined by the fit, together with the background fraction parameters $b$ and $f_{p}$.

The kinematic information is summarized by three loosely correlated observables: (a) the mass $m_{\pi \pi}$; (b) the signed momentum imbalance $\alpha=\left(1-p_{1} / p_{2}\right) q_{1}$, where $p_{1}\left(p_{2}\right)$ is the lower (higher) of the particle momenta, and $q_{1}$ is the sign of the charge of the particle of momentum $p_{1}$; (c) the scalar sum of particle momenta $p_{\text {tot }}=p_{1}+p_{2}$. The above variables allow evaluation of the invariant mass $m_{12}$ of a candidate for any mass assignment of the decay products $\left(m_{1}, m_{2}\right)$, using the equation

$$
\begin{aligned}
m_{12}^{2}= & m_{\pi \pi}^{2}-2 m_{\pi}^{2}+m_{1}^{2}+m_{2}^{2}-2 \sqrt{p_{1}^{2}+m_{\pi}^{2}} \sqrt{p_{2}^{2}+m_{\pi}^{2}} \\
& +2 \sqrt{p_{1}^{2}+m_{1}^{2}} \sqrt{p_{2}^{2}+m_{2}^{2}},
\end{aligned}
$$

where $p_{1}=\frac{1-|\alpha|}{2-|\alpha|} p_{\text {tot }}, p_{2}=\frac{1}{2-|\alpha|} p_{\text {tot }}$.

We used the mass sidebands in data $\left(m_{\pi \pi} \in\right.$ $\left.[5.00,5.12] \cup[5.6,6.2] \mathrm{GeV} / c^{2}\right)$ to obtain the kinematic distributions of backgrounds [16]. The mass distribution of the combinatorial background is parametrized by an exponential function, while the physics background is modeled by an ARGUS function [17] convoluted with a Gaussian resolution function. In order to ensure the reliability of the search for small signals in the vicinity of larger peaks, the shapes of the mass distributions assigned to each signal have been modeled in detail, including momentum dependence and non-Gaussian resolution tails from a full detector simulation, and the effects of soft photon radiation in the final state $[16,18]$. This resolution model describes very accurately the observed shape of the $D^{0} \rightarrow K^{-} \pi^{+}$ signal in a sample of $1.5 \times 10^{6} D^{*+} \rightarrow D^{0} \pi^{+}$decays, collected with a similar trigger selection. The $D^{*+} \rightarrow$ $D^{0} \pi^{+}$sample was also used to calibrate the $d E / d x$ response of the drift chamber to kaons and pions, using the charge of the pion from $D^{*+}$ decay to identify the $D^{0}$ decay products. The $d E / d x$ response of protons was determined from a sample of about $124000 \Lambda^{0} \rightarrow p \pi^{-}$decays. The model of the background allows for pion, kaon, proton, and electron components, whose fractions are determined by the fit. Muons are indistinguishable from pions with the available $10 \%$ fractional $d E / d x$ resolution and are therefore incorporated into the pion component.

From the signal fractions returned by the likelihood fit we calculate the signal yields shown in Table I. The significance of each signal is evaluated as the ratio of the yield observed in data, and its total uncertainty (statistical and systematic) as determined from a simulation where the size of that signal is set to zero. This evaluation assumes a Gaussian distribution of yield estimates, supported by the results obtained from repeated fits to simulated samples. We obtain significant signals for the $B_{s}^{0} \rightarrow K^{-} \pi^{+}$mode $(8.2 \sigma)$, and for the $\Lambda_{b}^{0} \rightarrow p \pi^{-}(6.0 \sigma)$ and $\Lambda_{b}^{0} \rightarrow p K^{-}$

TABLE I. Yields and significances of rare mode signals. The first quoted uncertainty is statistical, the second is systematic.

\begin{tabular}{lcc}
\hline \hline Mode & $N_{s}$ & Significance \\
\hline$B_{s}^{0} \rightarrow K^{-} \pi^{+}$ & $230 \pm 34 \pm 16$ & $8.2 \sigma$ \\
$B_{s}^{0} \rightarrow \pi^{+} \pi^{-}$ & $26 \pm 16 \pm 14$ & $<3 \sigma$ \\
$B^{0} \rightarrow K^{+} K^{-}$ & $61 \pm 25 \pm 35$ & $<3 \sigma$ \\
$\Lambda_{b}^{0} \rightarrow p K^{-}$ & $156 \pm 20 \pm 11$ & $11.5 \sigma$ \\
$\Lambda_{b}^{0} \rightarrow p \pi^{-}$ & $110 \pm 18 \pm 16$ & $6.0 \sigma$ \\
\hline \hline
\end{tabular}


TABLE II. Measured relative branching fractions of rare modes. The ratio $f_{\Lambda} / f_{d}$ is $p_{T}$ dependent [19], and is defined here as $f_{\Lambda} / f_{d}=\sigma\left(p \bar{p} \rightarrow \Lambda_{b}^{0} X ; p_{T}>6 \mathrm{GeV} / c,|\eta|<1\right) / \sigma\left(p \bar{p} \rightarrow B^{0} X ; p_{T}>6 \mathrm{GeV} / c,|\eta|<1\right)$. Absolute branching fractions were derived by normalizing to the current world-average value $\mathcal{B}\left(B^{0} \rightarrow K^{+} \pi^{-}\right)=(19.4 \pm 0.6) \times 10^{-6}$, and assuming the average values at high energy for the production fractions: $f_{s} / f_{d}=0.276 \pm 0.034$, and $f_{\Lambda} / f_{d}=0.230 \pm 0.052$ [20]. The first quoted uncertainty is statistical, the second is systematic.

\begin{tabular}{|c|c|c|}
\hline Mode & Relative $\mathcal{B}$ & Absolute $\mathcal{B}\left(10^{-6}\right)$ \\
\hline$B_{s}^{0} \rightarrow K^{-} \pi^{+}$ & $\frac{f_{s}}{f_{d}} \frac{\mathcal{B}\left(B_{s}^{0} \rightarrow K^{-} \pi^{+}\right)}{\mathcal{B}\left(B^{0} \rightarrow K^{+} \pi^{-}\right)}=0.071 \pm 0.010 \pm 0.007$ & $5.0 \pm 0.7 \pm 0.8$ \\
\hline$B_{s}^{0} \rightarrow \pi^{+} \pi^{-}$ & $\frac{f_{s}}{f_{d}} \frac{\mathcal{B}\left(B_{s}^{0} \rightarrow \pi^{+} \pi^{-}\right)}{\mathcal{B}\left(B^{0} \rightarrow K^{+} \pi^{-}\right)}=0.007 \pm 0.004 \pm 0.005$ & $0.49 \pm 0.28 \pm 0.36(<1.2$ at $90 \%$ C.L. $)$ \\
\hline$B^{0} \rightarrow K^{+} K^{-}$ & $\frac{\mathcal{B}\left(B^{0} \rightarrow K^{+} K^{-}\right)}{\mathcal{B}\left(B^{0} \rightarrow K^{+} \pi^{-}\right)}=0.020 \pm 0.008 \pm 0.006$ & $0.39 \pm 0.16 \pm 0.12(<0.7$ at $90 \%$ C.L. $)$ \\
\hline$\Lambda_{b}^{0} \rightarrow p K^{-}$ & $\frac{f_{\Lambda}}{f_{d}} \frac{\mathcal{B}\left(\Lambda_{b}^{0} \rightarrow p K^{-}\right)}{\mathcal{B}\left(B^{0} \rightarrow K^{+} \pi^{-}\right)}=0.066 \pm 0.009 \pm 0.008$ & $5.6 \pm 0.8 \pm 1.5$ \\
\hline$\Lambda_{b}^{0} \rightarrow p \pi^{-}$ & $\frac{f_{\Lambda}}{f_{d}} \frac{\mathcal{B}\left(\Lambda_{b}^{0} \rightarrow p \pi^{-}\right)}{\mathcal{B}\left(B^{0} \rightarrow K^{+} \pi^{-}\right)}=0.042 \pm 0.007 \pm 0.006$ & $3.5 \pm 0.6 \pm 0.9$ \\
\hline
\end{tabular}

$(11.5 \sigma)$ modes. No evidence is found for the modes $B_{s}^{0} \rightarrow$ $\pi^{+} \pi^{-}$or $B^{0} \rightarrow K^{+} K^{-}$, in agreement with expectations of significantly smaller branching fractions.

To avoid large uncertainties associated with production cross sections and absolute reconstruction efficiency, we measure all branching fractions relative to the $B^{0} \rightarrow$ $K^{+} \pi^{-}$mode. Results are listed in Table II. Frequentist upper limits [21] at the $90 \%$ C.L. are quoted for the unseen modes. For the measurement of $\Lambda_{b}^{0}$ branching fractions, the additional requirement $p_{T}\left(\Lambda_{b}^{0}\right)>6 \mathrm{GeV} / c$ was applied to allow easy comparison with other $\Lambda_{b}^{0}$ measurements at the Tevatron, which are only available above this threshold $[19,22]$. This additional requirement lowers the $\Lambda_{b}^{0}$ yields by about $20 \%$.

The raw fractions returned by the fit were corrected for the differences in selection efficiencies between different modes, which range from $8 \%$ to $40 \%$ for the measurements of $b$ mesons and $\Lambda_{b}^{0}$ branching fractions, respectively. These corrections were determined from detailed detector simulation, with the following exceptions that were measured from data: the momentum-averaged relative isolation efficiency between $B_{s}^{0}$ and $B^{0}, 1.00 \pm 0.03$, has been determined from fully reconstructed samples of $B_{s}^{0} \rightarrow$ $J / \psi \phi$, and $B^{0} \rightarrow J / \psi K^{* 0}$ decays [16]; the difference in efficiency for triggering on kaons and pions due to the different specific ionization in the COT $(\mathrm{a} \approx 5 \%$ effect $)$ was measured from a sample of $D^{+} \rightarrow K^{-} \pi^{+} \pi^{+}$decays triggered on two tracks, using the unbiased third track [23]. Possible differences in efficiency of the isolation requirement between $B^{0}$ and $\Lambda_{b}^{0}$, and in the trigger efficiency between kaons and protons, were taken into account in the systematic uncertainties.

The dominant contributions to the systematic uncertainty are the uncertainty on the combinatorial background model and the uncertainty on the $d E / d x$ calibration and parametrization. Other contributions come from trigger efficiencies, physics background shape and kinematics, $b$ hadron masses and lifetimes, and the possible polarization of $\Lambda_{b}^{0}$ decays. Details of the systematic uncertainties can be found in Ref. [16].
Absolute branching fractions are also quoted in Table II, by normalizing to world-average values of production fractions and $\mathcal{B}\left(B^{0} \rightarrow K^{+} \pi^{-}\right)$[20]. The branching fraction measured for the $B_{s}^{0} \rightarrow K^{-} \pi^{+}$mode is consistent with the previous upper limit $\left(<5.6 \times 10^{-6}\right.$ at $90 \%$ C.L. $)$, based on a subsample of the current data [3]. This agrees with the prediction in Ref. [24], but it is lower than most other predictions $[5,6,25]$. The $B_{s}^{0} \rightarrow \pi^{+} \pi^{-}$upper limit improves and supersedes the previous best limit [3]. The present measurement of $\mathcal{B}\left(B^{0} \rightarrow K^{+} K^{-}\right)$is in agreement with other existing measurements and has a similar resolution [20], but the resulting upper limit is weaker due to the observed central value. The sensitivity to both $B^{0} \rightarrow$ $K^{+} K^{-}$and $B_{s}^{0} \rightarrow \pi^{+} \pi^{-}$is now close to the upper end of the theoretically expected range [5-7,26]. We also report the first branching fraction measurements of charmless $\Lambda_{b}$ decays. They are significantly lower than the previous upper limit of $2.3 \times 10^{-5}$ [27], and in reasonable agreement with predictions [9], thus excluding the possibility of large $\left[O\left(10^{2}\right)\right]$ enhancements from $R$-parity violating supersymmetric scenarios [28]. Their ratio can be determined directly from our data with greater accuracy than the individual values. For this purpose, the additional $p_{T}>$ $6 \mathrm{GeV} / c$ requirement is not necessary, and we can exploit the full sample size, obtaining $\mathcal{B}\left(\Lambda_{b}^{0} \rightarrow p \pi^{-}\right) / \mathcal{B}\left(\Lambda_{b}^{0} \rightarrow\right.$ $\left.p K^{-}\right)=0.66 \pm 0.14 \pm 0.08$, in good agreement with the predicted range $0.60-0.62$ [9].

In summary, we have searched for rare charmless decay modes of neutral $b$ hadrons into pairs of charged hadrons in $\mathrm{CDF}$ data. We report the first observation of the modes $B_{s}^{0} \rightarrow K^{-} \pi^{+}, \Lambda_{b}^{0} \rightarrow p \pi^{-}$, and $\Lambda_{b}^{0} \rightarrow p K^{-}$, and measure their relative branching fractions. We set upper limits on the unobserved modes $B^{0} \rightarrow K^{+} K^{-}$and $B_{s}^{0} \rightarrow \pi^{+} \pi^{-}$.

We thank the Fermilab staff and the technical staffs of the participating institutions for their vital contributions. This work was supported by the U.S. Department of Energy and National Science Foundation; the Italian Istituto Nazionale di Fisica Nucleare; the Ministry of Education, Culture, Sports, Science and Technology of Japan; the Natural Sciences and Engineering Research 
Council of Canada; the National Science Council of the Republic of China; the Swiss National Science Foundation; the A.P. Sloan Foundation; the Bundesministerium für Bildung und Forschung, Germany; the Korean Science and Engineering Foundation and the Korean Research Foundation; the Science and Technology Facilities Council and the Royal Society, U.K.; the Institut National de Physique Nucleaire et Physique des Particules/CNRS; the Russian Foundation for Basic Research; the Ministerio de Ciencia e Innovación, and Programa Consolider-Ingenio 2010, Spain; the Slovak R\&D Agency; and the Academy of Finland.

${ }^{\mathrm{a} D e c e a s e d .}$

${ }^{\mathrm{b}}$ Visitor from University of Massachusetts Amherst, Amherst, MA 01003, USA.

${ }^{\mathrm{c}}$ Visitor from Universiteit Antwerpen, B-2610 Antwerp, Belgium.

${ }^{\mathrm{d}}$ Visitor from University of Bristol, Bristol BS8 1TL, United Kingdom.

${ }^{\mathrm{e}}$ Visitor from Chinese Academy of Sciences, Beijing 100864, China.

${ }^{\mathrm{f}}$ Visitor from Istituto Nazionale di Fisica Nucleare, Sezione di Cagliari, 09042 Monserrato (Cagliari), Italy.

${ }^{\mathrm{g}}$ Visitor from University of California Irvine, Irvine, CA 92697, USA.

${ }^{\mathrm{h}}$ Visitor from University of California, Santa Cruz, Santa Cruz, CA 95064, USA.

${ }^{\mathrm{i}}$ Visitor from Cornell University, Ithaca, NY 14853, USA.

${ }^{j}$ Visitor from University of Cyprus, Nicosia CY-1678, Cyprus.

${ }^{\mathrm{k}}$ Visitor from University College Dublin, Dublin 4, Ireland.

${ }^{1}$ Visitor from University of Edinburgh, Edinburgh EH9 3JZ, United Kingdom.

${ }^{\mathrm{m}}$ Visitor from Universidad Iberoamericana, Mexico D.F., Mexico.

${ }^{\mathrm{n}}$ Visitor from Queen Mary, University of London, London, E1 4NS, United Kingdom.

${ }^{\circ}$ Visitor from University of Manchester, Manchester M13 9PL, United Kingdom.

${ }^{\mathrm{p}}$ Visitor from Nagasaki Institute of Applied Science, Nagasaki, Japan.

${ }^{\mathrm{q}}$ Visitor from University of Notre Dame, Notre Dame, IN 46556, USA.

${ }^{\mathrm{r}}$ Visitor from University de Oviedo, E-33007 Oviedo, Spain.

${ }^{\mathrm{s}}$ Visitor from Texas Tech University, Lubbock, TX 79409 , USA.

${ }^{t}$ Visitor from IFIC (CSIC-Universitat de Valencia), 46071 Valencia, Spain.

"Visitor from University of Virginia, Charlottesville, VA 22904, USA.

${ }^{\mathrm{v}}$ Visitor from Bergische Universität Wuppertal, 42097 Wuppertal, Germany.

${ }^{\mathrm{w}}$ On leave from J. Stefan Institute, Ljubljana, Slovenia.
[1] B. Aubert et al. (BABAR Collaboration), Phys. Rev. D 75, 012008 (2007); S.-W. Lin et al. (Belle Collaboration), Phys. Rev. Lett. 99, 121601 (2007); A. Bornheim et al. (CLEO Collaboration), Phys. Rev. D 68, 052002 (2003).

[2] R. Fleischer, Phys. Lett. B 459, 306 (1999); A. Soni and D. A. Suprun, Phys. Rev. D 75, 054006 (2007).

[3] A. Abulencia et al. (CDF Collaboration), Phys. Rev. Lett. 97, 211802 (2006).

[4] M. Gronau and J. L. Rosner, Phys. Lett. B 482, 71 (2000).

[5] J.-F. Sun, G.-H. Zhu, and D.-S. Du, Phys. Rev. D 68, 054003 (2003).

[6] M. Beneke and M. Neubert, Nucl. Phys. B675, 333 (2003).

[7] A. Ali et al., Phys. Rev. D 76, 074018 (2007); X.-Q. Yu, Y. Li, and C.-D. Lu, Phys. Rev. D 71, 074026 (2005); 72, 119903(E) (2005).

[8] A. J. Buras, R. Fleischer, S. Recksiegel, and F. Schwab, Nucl. Phys. B697, 133 (2004).

[9] R. Mohanta, A. K. Giri, and M. P. Khanna, Phys. Rev. D 63, 074001 (2001).

[10] Throughout this Letter, $C$ conjugate modes are implied and branching fractions indicate $C P$ averages.

[11] D. Acosta et al. (CDF Collaboration), Phys. Rev. D 71, 032001 (2005); A. Sill (CDF Collaboration), Nucl. Instrum. Methods Phys. Res., Sect. A 447, 1 (2000); A. Affolder et al., Nucl. Instrum. Methods Phys. Res., Sect. A 453, 84 (2000); T. Affolder et al., Nucl. Instrum. Methods Phys. Res., Sect. A 526, 249 (2004).

[12] CDF II uses a cylindrical coordinate system in which $\phi$ is the azimuthal angle, $r$ is the radius from the nominal beam line, and $z$ points in the proton beam direction, with the origin at the center of the detector. The transverse plane is the plane perpendicular to the $z$ axis

[13] E. Thomson et al., IEEE Trans. Nucl. Sci. 49, 1063 (2002); R. Downing et al., Nucl. Instrum. Methods Phys. Res., Sect. A 570, 36 (2007); B. Ashmanskas et al., Nucl. Instrum. Methods Phys. Res., Sect. A 518, 532 (2004).

[14] Isolation is defined as $I_{B}=p_{T}(B) /\left(p_{T}(B)+\sum_{i} p_{T i}\right)$, where $p_{T}(B)$ is the transverse momentum of the $B$ candidate, and the sum runs over all other tracks within a cone of radius 1 , in $\eta-\phi$ space around the $B$ flight direction.

[15] G. Punzi, eConf C030908, MODT002 (2003).

[16] M. J. Morello, Ph.D. thesis, Scuola Normale Superiore, Pisa [Fermilab Report No. FERMILAB-THESIS-2007-57, 2007].

[17] Defined as $m_{\pi \pi} e^{-c_{A}\left(m_{\pi \pi} / m_{A}\right)^{2}} \sqrt{1-\left(m_{\pi \pi} / m_{A}\right)^{2}}$, for $m_{\pi \pi}<$ $m_{A}$. The cutoff $m_{A}$ and the coefficient $c_{A}$ were free parameters in our fit. See H. Albrecht et al. (ARGUS Collaboration), Phys. Lett. B 241, 278 (1990).

[18] E. Baracchini and G. Isidori, Phys. Lett. B 633, 309 (2006).

[19] T. Aaltonen et al. (CDF Collaboration), Phys. Rev. D 79, 032001 (2009).

[20] C. Amsler et al., Phys. Lett. B 667, 1 (2008).

[21] G. J. Feldman and R. D. Cousins, Phys. Rev. D 57, 3873 (1998).

[22] A. Abulencia et al. (CDF Collaboration), Phys. Rev. Lett. 98, 122002 (2007). 
[23] D. Acosta et al. (CDF Collaboration), Phys. Rev. Lett. 94, 122001 (2005).

[24] A. R. Williamson and J. Zupan, Phys. Rev. D 74, 014003 (2006); 74, 039901(E) (2006).

[25] C.-W. Chiang, M. Gronau, and J. L. Rosner, Phys. Lett. B 664, 169 (2008).
[26] Y. Li, C.-D. Lu, Z.-J. Xiao, and X.-Q. Yu, Phys. Rev. D 70, 034009 (2004).

[27] D. Acosta et al. (CDF Collaboration), Phys. Rev. D 72, 051104 (2005).

[28] R. Mohanta, Phys. Rev. D 63, 056006 (2001). 